\title{
Editor's Message to Special Issue of Information Systems
}

\author{
Yoshiaki MatsuZawa $^{1, a)}$
}

Recently in information and communication technology field, applications of state-of-the-art technologies such as AI (Artificial Intelligence), big data analysis have attracted attention in development of information systems. However, the fundamental role of information systems: "collect, operate, store, and distribute information in organization", is still not necessary to be changed. Actually, advances in technology such as AI promotes further discussion about how we can harmonize technologies and human/organizational needs in a design of information systems. Since 2005, the Special Interest Group on Information Systems (SIG-IS), has planned a special issue of papers on information systems every year, and selected quality papers for publication in order to contribute to the dissemination of and awareness-raising about information systems.

As with previous special issues, we have invited papers with a wide range of subjects for this special issue, including: theory and practice regarding the analysis, design, construction, operation, and use of information systems; interrelations between information systems and people, organizations, and society; knowledge obtained from system development in various organizations; and proposals for new information systems which recognize information needs.

There were 10 papers submitted, a lower number than originally expected, but they covered a variety of application areas. We intended to have an acceptance rate of $50 \%$, but with seven published papers, the actual figure came in below the target at $30 \%$. Nevertheless, I believe we were able to publish papers of sufficient quality.

The topics of the accepted three papers are: (1) design of a middleware for smart home network, (2) design of a system for sharing teaching information in education, and (3) design of a system for performance analysis in sport. Each paper carefully discusses their organizational issues and design factors toward a solution, so that we concluded that we could accept appropriate papers in accordance with our original aims.

Papers on information systems cover an extremely broad range of subject matter, and partly for that reason, it is difficult to evaluate the effectiveness of papers and ensure their accuracy. As one attempt to deal with such issues, the SIG-IS has released guidelines for quantitative and qualitative evaluation indicating techniques for evaluating effectiveness of information systems. In this special issue, we strived for uniformity in peer review standards by asking reviewers to read these documents as part of their review of qualitative research papers. In addition, the SIG-IS is

\footnotetext{
Aoyama Gakuin University, Kanagawa 252-5258, Japan
}

a) matsuzawa@si.aoyama.ac.jp working to improve the quality and quantity of submitted papers by, for example, planning Q\&A sessions that are a little longer at meetings to present research, with the intention of also promoting submission to the special issue.

This time too, many case studies of development were submitted, but paper effectiveness is not always described based on a proper quantitative and qualitative evaluation, and going forward, we would like to continue examining approaches enabling more accessible learning of quantitative and qualitative evaluation methods for papers on information systems. We also expect these activities to contribute to the development of information systems.

Finally, I would like to express our sincere gratitude to the journal editorial committee which provided the opportunity for this special issue, the special issue editorial committee which carried out careful review in a short review period, all of the reviewers, and the person's in charge at the IPSJ who provided various types of support including schedule management.

\section{The Editorial Committee}

- Editor in-Chief:

Yoshiaki Matsuzawa (Aoyama Gakuin University)

- Editorial Board Member:

Yoshio Kakizaki (Tokyo Denki University)

\section{- Editorial Committee Members:}

Akihiro Abe (Iwate Prefectural University)

Michiko Oba (Future University Hakodate)

Susumu Kanemune (Osaka Electro-Communication University)

Kiminobu Kodama (Information Systems Institute, Ltd.)

Akira Goto (Tama University)

Makoto Tachikawa (Muroran Institute of Technology)

Hidekazu Tsuji (Non-Profit Organization Study Group on M2M)

Masaki Tomisawa (Maebashi Institute of Technology)

Michinori Hatayama (Kyoto University)

Masahiro Hiji (Hitachi Solutions East Japan, Ltd.)

Hidemi Fukada (Otaru University of Commerce)

Masami Honda (Tokyo Institute of Technology)

Hiroshi Maruyama (Aoyama Gakuin University)

Hiroyoshi Watanabe (Teikyo University) 